\title{
Feature Extraction of an Image by Using Adaptive Filtering and Morpological Segmentation
}

\author{
Anil kumar. $\mathrm{M}^{1}$ Fahimuddin shaik ${ }^{2}$ Abdul Rahim. $\mathrm{B}^{3}$ \\ ${ }^{I}$ Post graduate student, Dept. of E.C.E, A.I.T.S, Rajampet, AP. \\ ${ }^{2}$ Assistant professor, Dept. of E.C.E AITS, Rajampet, A.P. \\ ${ }^{3}$ H.O.D, Dept. of E.C.E AITS, Rajampet, A.P.
}

\begin{abstract}
For enhancing an image various enhancement schemes are used which includes gray scale manipulation, filtering and Histogram Equalization, Where Histogram equalization is one of the well known image enhancement technique. It became a popular technique for contrast enhancement because it is simple and effective. The basic idea of Histogram Equalization method is to remap the gray levels of an image.

Here using morphological segmentation we can get the segmented image. Morphological reconstruction is used to segment the image.

Comparative analysis of different enhancement and segmentation will be carried out. This comparison will be done on the basis of subjective and objective parameters. Subjective parameter is visual quality and objective parameters are Area, Perimeter, Min and Max intensity, Avg Voxel Intensity, Std Dev of Intensity, Eccentricity, Coefficient of skewness, Coefficient of Kurtosis, Median intensity, Mode intensity.
\end{abstract}

Keywords: Histogram Equalization, Segmentation, Morphological Reconstruction .

\section{Introduction:}

Medical image enhancement technologies have become popular since advanced medical equipments were used in the medical field. Image processing can be defined as a process that transforms a degraded image to another image of better quality in order to facilitate its posterior interpretation. Image processing techniques can be then applied in order to facilitate the interpretation of images by further using computer vision algorithms. Therefore, in this context, image processing can be considered to be a preprocessing stage for computer vision [3]. The process involved in making an image more interpretable for particular application is image enhancement.

Image enhancement is basically improving the interpretability or perception of information in images for human viewers and providing 'better' input for other automated image processing techniques. The principal objective of image enhancement is to modify attributes of an image to make it more suitable for a given task and a specific observer. During this process, one or more attributes of the image are modified. The choice of attributes and the way they are modified are specific to a given task. Moreover, observer-specific factors, such as the experience, will introduce a great deal of subjectivity into the choice of image enhancement methods. There exist many techniques that can enhance a digital image without spoiling it. The enhancement methods can broadly be divided in to the following two categories:

1. Spatial Domain Methods

2. Frequency Domain Methods

In spatial domain techniques [5], we directly deal with the image pixels. The pixel values are manipulated to achieve desired enhancement. In frequency domain methods, the image is first transferred in to frequency domain. It means that, the Fourier Transform of the image is computed first. All the enhancement operations are performed on the Fourier transform of the image and then the Inverse Fourier transform is performed to get the resultant image. These enhancement operations are performed in order to modify the image brightness, contrast or the distribution of the grey levels. As a consequence the pixel value (intensities) of the output image will be modified according to the transformation function applied on the input values.

Morphological image processing is a collection of non-linear operations related to the shape or morphology of features in an image. Morphological operations rely only on the relative ordering of pixel values, not on their numerical values, and therefore are especially suited to the processing of binary images. Morphological operations can also be applied to grayscale images such that their light transfer functions are unknown and therefore their absolute pixel values are of no or minor interest.

In this paper we will have Literature survey of image enhancement and image segmentation, proposed method, experimental results, statistical analysis of results; finally we will end with our conclusion. 


\section{Background:}

Before going to details of the proposed method, we present some background topics of image enhancement which include CLAHE and Image segmentation.

\section{A. Image Enhancement:}

Image enhancement [1] is the simplest and most appealing areas of digital image processing. Basically, the main idea behind enhancement techniques [3] is to bring out detail that is obscured, or simply to highlight certain features of interest in an image. We have proposed the following CLAHE, gamma correction techniques to enhance the medical images.

\section{B. Contrast Limited Adaptive Histogram Equalization (CLAHE) :}

Contrast Limited Adaptive Histogram Equalization differs from ordinary adaptive histogram equalization in its contrast limiting. This feature can also be applied to global histogram equalization, giving rise to contrast limited histogram equalization, which is rarely used in practice. In the case of contrast limited histogram equalization, the contrast limiting procedure has to be applied for each neighborhood from which a transformation function is derived. Contrast limited histogram equalization was developed to prevent the over amplification of noise, which is a problem in adaptive histogram equalization.

\section{Image segmentation:}

Segmentation [7] subdivides an image into its constituent regions or objects. The level to which the subdivision is carried depends. That is, segmentation should stop when the objects of interest in an application have been isolated .Segmentation of nontrivial images is one of the most difficult tasks in image processing. Segmentation accuracy determines the eventual success or failure of computerized analysis procedures. For this reason, considerable care should be taken to improve the probability of rugged segmentation. In some situations, such as industrial inspections applications, at least some measure of control over the environment is possible in times. The experienced image processing system designer invariably pays considerable attention to such opportunities. In other applications, such as autonomous target acquisition, the system designers have no control of the environment. Then the usual approach is to focus on selecting the types of sensors most likely to enhance the objects of interest while diminishing the contribution of irrelevant image details. A good example is the use of infrared imaging by military to detect objects with strong heat signatures, such as equipment and troops in motion.

Image segmentation algorithms generally are based on one of two basic properties of intensity values: discontinuity and similarity. In the first category, the approach is to partition an image based an abrupt change in intensity, such as edges in an image. The principal approaches in the second category are based on partitioning an image into regions that are similar according to set of predefined criteria. Thresholding, region growing, and region splitting and merging are methods in this category. There are several techniques for detecting the three basic types of gray-level discontinuities in an digital image: point, line and edge detection.

\section{Morphological Reconstruction:}

Reconstruction [8] is a morphological transformation involving two images and a structuring element (instead of a single image and structuring element). One image, the marker, is the starting point for the transformation. The other image, the mask, constrains the transformation. The structuring element used defines connectivity. In this section we use 8-connectivity (the default), which implies that B in the following discussion is a $3 \times 3$ matrix of $1 \mathrm{~s}$, with the center defined at coordinates $(2,2)$. In this section we deal with binary images; If $G$ is the mask and $F$ is the marker, the reconstruction of $G$ from $F$, denoted $R_{G}(\mathrm{~F})$, is defned by the following iterative procedure:

1. Initialize h1 to be the marker image, $\mathrm{F}$.

2. Create the structuring element: $\mathrm{B}=$ ones (3).

3. Repeat: $h_{k+1}=\left(h_{k} \oplus B\right) \cap G$

4. $R_{G}(\mathrm{~F})=h_{k+1}$.

$$
\text { until } h_{k+1}=h_{k} \text {. }
$$

Marker $\mathrm{F}$ must be a subset of $\mathrm{G}: \quad \mathrm{F} \subseteq G$

\section{E. Opening and Closing by Reconstruction:}

In morphological opening, erosion typically removes small objects, and the sub-sequent dilation tends to restore the shape of the objects that remain. However, the accuracy of this restoration depends on the similarity between the shapes and the structuring element. The method discussed in this section, opening by reconstruction, restores the original shapes of the objects that remain after erosion. The opening by reconstruction of an image $\mathrm{G}$ using structuring element $\mathrm{B}$, is defined as $R_{G}(G \ominus B)$ 


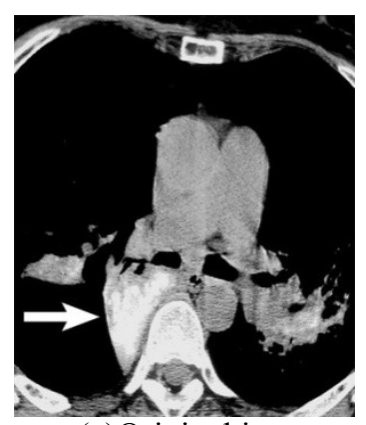

(a)Original image

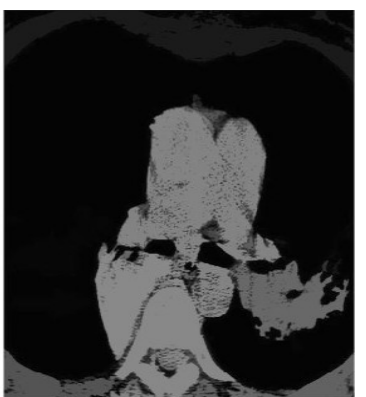

(c) Opening by Reconstruction (d) closing the image

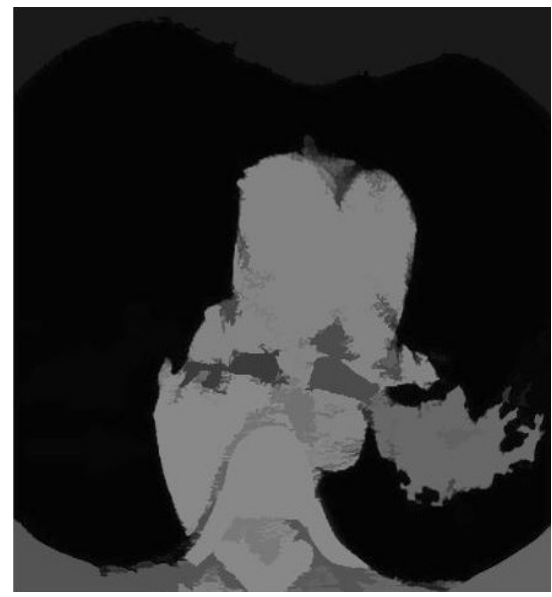

(e) Closing by Reconstruction

III. Proposed Work:

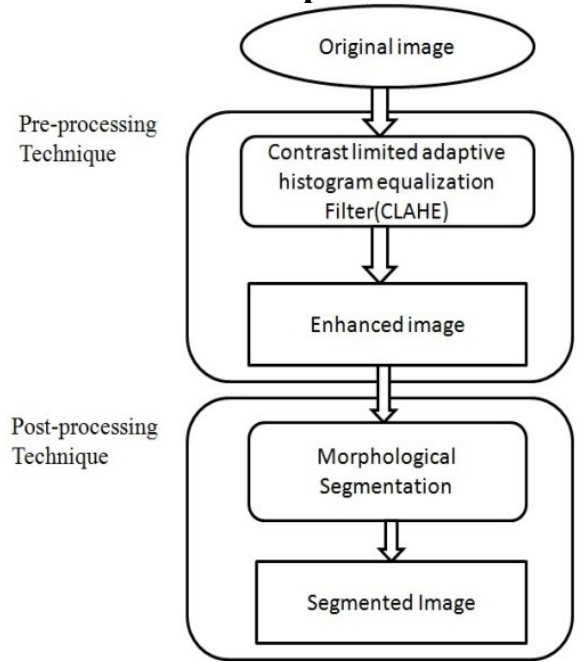

Figure . 1 : Block Diagram of Proposed work 
The proposed method is used to enhance and segment the medical image. We use the Contrast limited adaptive Histogram Equalization (CLAHE) to enhance the medical images, and Morphological techniques for segment the Enhanced medical image.

\section{Experimental Results :}

Here we can use some medical image modalities like MRI, CT scan, ECG etc. For example here we are using an image of Talc embolism in a 26-year-old woman. The patient had a 4-year history of heroin and methadone abuse which is Thin-section (1.5-mm collimation) CT scan (mediastinal window) obtained at the subcarinal level shows coalescent areas of increased attenuation (progressive massive fibrosis) posteriorly in both lungs. Note also the areas of high attenuation within the masses (arrow), a finding that suggests talc deposition. Figure 2.(a,b,c,d) shows experimental results. Figs. 2.a is original image, Figs. 2.b is enhanced image via CLAHE, Figs.2.c is Segmented Image by Reconstruction and Fig.2.d. is superimposed on original image.

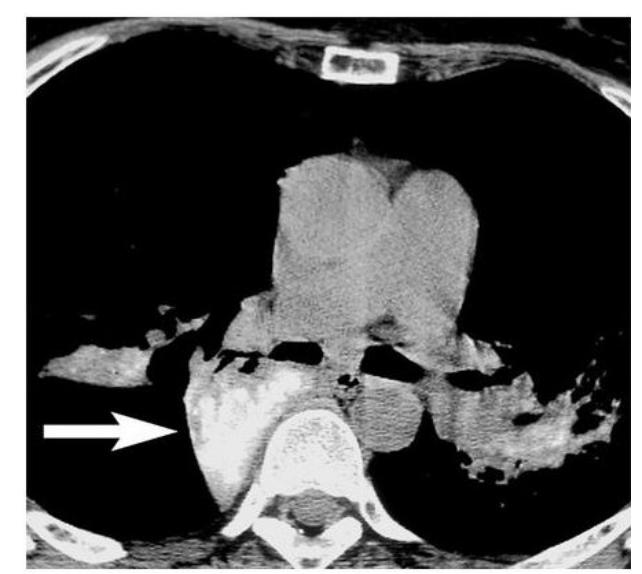

Figure.2(a) Original CT Image

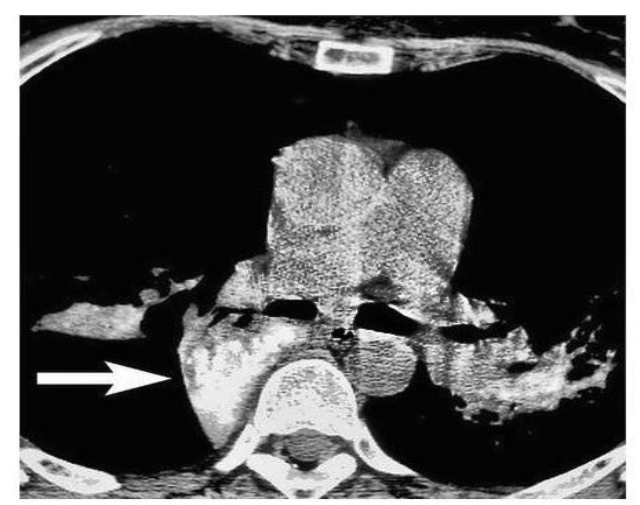

Figure.2(b) Enhanced Image by CLAHE

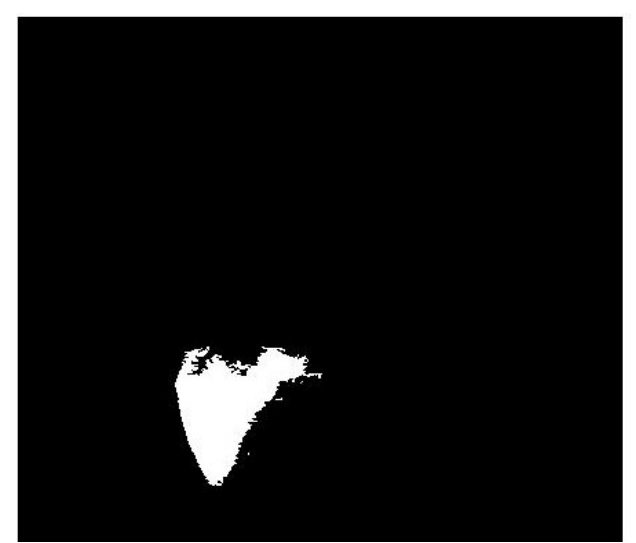

Figure.2(c) Segmented Image by Reconstruction 


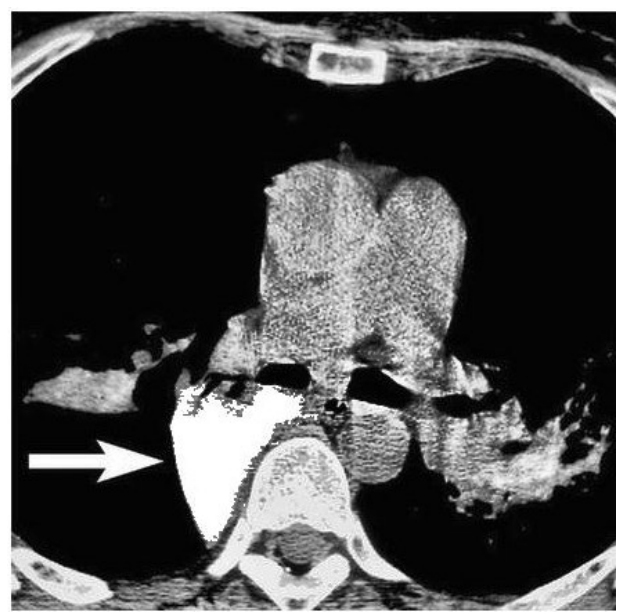

Figure.2(d) superimposed on original image

\section{Statistical Analysis :}

The performance of the proposed method was rigorously evaluated using quality metrics like Area, Perimeter, Min and Max intensity, Avg Voxel Intensity, Std Dev of Intensity, Eccentricity, Coefficient of skewness, Coefficient of Kurtosis, Median intensity, Mode intensity.

Table1: Quality Assessment metrics for original image

\begin{tabular}{|l|l|}
\hline Area & 4975 \\
\hline Perimeter & 325.0554 \\
\hline Min Intensity & 45 \\
\hline Max Intensity & 245 \\
\hline Avg Voxel Intensity & 212.1672 \\
\hline Std Dev of Intensity & 25.5892 \\
\hline Eccentricity & 0.7844 \\
\hline Coefficient of skewness & -1.3619 \\
\hline Coefficient of kurtosis & 5.0113 \\
\hline Median Intensity & 224 \\
\hline Mode Intensity & 223 \\
\hline
\end{tabular}

Table2: Quality Assessment metrics for segmented image.

\begin{tabular}{|l|l|}
\hline Area & 4317 \\
\hline Perimeter & 314.6178 \\
\hline Min Intensity & 0 \\
\hline Max Intensity & 255 \\
\hline Avg Voxel Intensity & 238.1828 \\
\hline Std Dev of Intensity & 46.5425 \\
\hline Eccentricity & 0.7933 \\
\hline Coefficient of skewness & -3.0504 \\
\hline Coefficient of kurtosis & 11.338 \\
\hline Median Intensity & 255 \\
\hline Mode Intensity & 255 \\
\hline
\end{tabular}

By observing Area, Perimeter, Min and Max intensity, Avg Voxel Intensity, Std Dev of Intensity, Eccentricity, Coefficient of skewness, Coefficient of Kurtosis, Median intensity, Mode intensity the changes occurred after processing is easily revealed.

\section{Conclusion :}

In this paper we proposed image enhancement method and image segmentation using Morphological techniques.

By observing all of the above quality assessment metrics for "CT of Lungs" image, we can conclude that CLAHE enhancement method is having less noise and having more contrast. The enhanced image is segmented by using Morphological techniques. By the segmentation we can get the segmented image and it is superimposed on the original image, to get the perfect outlined structure of the talc deposition in the image. 


\section{References:}

[1]. Rajesh Garang ,Bhavna Mittal, Sheetal Garg "Histogram Equalization Techniques For Image Enhancement" ,IJECT vol. 2,Issue1, march2011.

[2]. H. Hassanpour* and S. Asadi Amiri , "Image Quality Enhancement Using Pixel-Wise Gamma Correction Via Svm Classifier" September $14,2011$.

[3]. K. Ikeuchi; C. Schnörr; M. Hebert; C. Schmid,"International Journal of Computer Vision"ISSN: 0920-5691,Journal no. 11263.

[4]. Raman Maini and Himanshu Aggarwal,"A Comprehensive Review of Image Enhancement Techniques " JOURNAL OF COMPUTING, VOLUME 2, ISSUE 3, MARCH 2010, ISSN 2151-9617

[5]. P.Nithyanandam, T.Ravichandran, N.M.Santron \& E.Priyadarshini ,"A Spatial Domain Image Steganography Technique Based on Matrix Embedding and Huffman Encoding" International Journal of Computer Science and Security (IJCSS), Volume (5) : Issue (5) : 2011

[6]. Etta D.Pisano,Shuquan Zong, Bradley M.Hemminger,Marla DeLuca,R.Eugene Johnston,KeithMuller,M.Patricia Braeuning and Stephen M.Pizer,"Contrast Limited Adaptive Histogram Equalization image processing to improve the detection of simulated spiculations in dense mammograms"Journal of Digital Imaging November 1998, Volume 11, Issue 4, pp 193 -200

[7]. Biplab Banerjee, Tanusree Bhattacharjee \& Nirmalya Chowdhury "Color Image Segmentation Technique Using "Natural Grouping" of Pixels"International Journal of Image Processing(IJIP), Volume(4): Issue(4).

[8]. David Valencia and Antonio Plaza,"efficient implementation of morphological opening and closing by reconstruction on multi-core parallel systems",IEEE Xplore 2009,Page 1-4, E-ISBN : 978-1-4244-4687-2

[9]. Www.mathworks.in.

[10]. www.mipav.cit.nih.gov

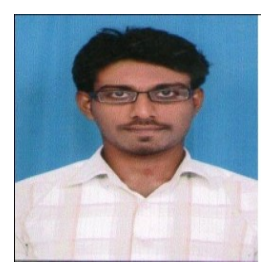

M.Anilkumar born in Nellore, A.P, India in 1989. He received B.Tech Degree in Electronics \&Communication Engg. From J.N.T.University, Anantapur, India. Presently he is pursuing M.Tech (DECS) from Annamacharya Institute of Technology \& Sciences, Rajampet, A.P., India.His research interests include Biomedical engineering, Image Processing and Communication Systems.

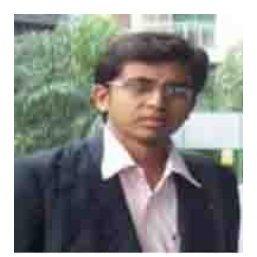

Fahimuddin Shaik did his B. Tech and M.Tech in Electronics \& Communication Engineering (ECE) from JNT University, Hyderabad, India. He is currently working towards a $\mathrm{PhD}$ in biomedical image processing. He is an assistant professor in the Department of ECE at the Annamacharya Institute of Technology \& Sciences (an Autonomous Institute), in Rajampet, Andhra Pradesh. He is also the Academic Council Member of the Institute. His research interests include signal processing, time series analysis, and biomedical image processing. He has presented many research papers at national and international conferences. He ha s authored a book "MEDICAL IMAGING IN DIABETES, VOL 1- A Technical Approach", Cinnamonteal Publishing, December 2011.

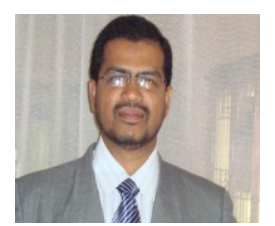

B Abdul Rahim born in Guntakal, A.P, India in 1969. He received the B.E in Electronics \& Communication Engineering from Gulbarga University in 1990. M.Tech (Digital Systems \&Computer Electronics) from Jawaharlal Nehru Technological University in 2004. He is currently working towards Ph.D. degree from JNT University, Anantapur. He has published papers in international journals and conferences. He is a member of professional bodies like IEEE,EIE, ISTE, IACSIT, IAENG etc. His research interests include Fault Tolerant Systems, Embedded Systems and parallel processing. He achieved "Best Teacher Award" for his services by Lions Club, Rajampet. 